\title{
ON $P$-I-OPEN SETS
}

\author{
Jeong Gi Kang and Chang Su Kim
}

\begin{abstract}
The notions of pre-local function, semi-local functions and $\alpha$-local functions with respect to a topology and an ideal are introduced, and several properties are investigated. Also, the concept of $P$-I -open sets and $P$ - $\mathcal{I}$-closed sets in ideal topological spaces are discussed. Relations between $\mathcal{I}$-open sets and $P$ - $\mathcal{I}$-open sets are provided, and several properties related to $P$-I $\mathcal{I}$-open sets, pre-local functions, semi-local functions and $\alpha$-local functions with respect to a topology and an ideal are investigated.
\end{abstract}

\section{Introduction}

In 1990, D. Janković, and T.R. Hamlett have introduced the notion of $\mathcal{I}$-open sets in topological spaces. Since then, several kinds of $\mathcal{I}$ openness, that is, (weakly) semi-I-I-open set, $\delta$-I -open sets, $\beta$-I $\mathcal{I}$-open sets, $\alpha$-I -open sets, $b$ - $\mathcal{I}$-open sets, (weakly) pre- $\mathcal{I}$-open sets, etc. are introduced, and several properties and relations are investigated (see $[1,2,6,8,9,10,7,19,23])$.

In this paper, we first introduce the notions of pre-local function, semi-local function and $\alpha$-local function with respect to a topology and an ideal, and several properties are investigated. We next introduce the concept of $P$-I-open set and $P$-I-closed set in ideal topological spaces, and investigates related properties. We discuss relations between $\mathcal{I}$ open sets and $P$-I-open sets. Finally we introduce the notion of $P$-*closure, and investigates many properties related to $P$ - $\mathcal{I}$-open set, prelocal function, semi-local function and $\alpha$-local function with respect to a topology and an ideal.

Received April 8, 2009. Accepted July 13, 2009. (Top/HMJ080615-P-I-open set.tex)

2000 Mathematics Subject Classification. 54A40.

Key words and phrases. Ideal topological spaces, pre-local (semi-local, $\alpha$-local) functions with respect to a topology and an ideal, $P$ - $\mathcal{I}$-open (closed) sets, . 


\section{Preliminaries}

Through this paper, $(X, \tau)$ and $(Y, \kappa)$ (simply $X$ and $Y$ ) always mean topological spaces. A subset $A$ of $X$ is said to be semi-open [20] (respectively, $\alpha$-open [21] and pre-open [18]) if $A \subset \mathrm{Cl}(\operatorname{Int}(A))$ (respectively, $A \subset \operatorname{Int}(\mathrm{Cl}(\operatorname{Int}(A)))$ and $A \subset \operatorname{Int}(\mathrm{Cl}(A)))$. The complement of a preopen set (respectively, an $\alpha$-open set and a semi-open set) is called a pre-closed set (respectively, an $\alpha$-closed set and a semi-closed set). The intersection of all pre-closed sets (respectively, $\alpha$-closed sets and semi-closed sets) containing $A$ is called the pre-closure (respectively, $\alpha$ closure and semi-closure) of $A$, denoted by $p \mathrm{Cl}(A)$ (respectively, $\alpha \mathrm{Cl}(A)$ and $s \mathrm{Cl}(A)$ ). A subset $A$ is also pre-closed (respectively, $\alpha$-closed and semi-closed) if and only if $A=p \operatorname{Cl}(A)$ (respectively, $A=\alpha \mathrm{Cl}(A)$ and $A=s \mathrm{Cl}(A)$ ). We denote the family of all pre-open sets (respectively, $\alpha$-open sets and semi-open sets) of $(X, \tau)$ by $\tau^{p}$ (respectively, $\tau^{\alpha}$ and $\left.\tau^{s}\right)$.

An ideal is defined as a nonempty collection $\mathcal{I}$ of subsets of $X$ satisfying the following two conditions.

1. If $A \in \mathcal{I}$ and $B \subset A$, then $B \in \mathcal{I}$. (heredity)

2. If $A \in \mathcal{I}$ and $B \in \mathcal{I}$, then $A \cup B \in \mathcal{I}$. (finite additivity)

An ideal topological space is a topological space $(X, \tau)$ with an ideal $\mathcal{I}$ on $X$, and it is denoted by $(X, \tau, \mathcal{I})$. For a subset $A \subset X$, the set

$$
A^{*}(\tau, \mathcal{I})=\{x \in X: U \cap A \notin \mathcal{I} \text { for each } U \in \tau(x)\}
$$

is called the local function of $A$ with respect to $\tau$ and $\mathcal{I}$, where

$$
\tau(x)=\{U \in \tau: x \in U\} .
$$

We will use $A^{*}$ and/or $A^{*}(\mathcal{I})$ instead of $A^{*}(\tau, \mathcal{I})$. Given ideal topological space $(X, \tau, \mathcal{I})$, the $*$-topology, written as $\tau^{*}(\mathcal{I})$ or written simply as $\tau^{*}$, on $X$ is defined to be a topology with a basis

$$
\beta(\mathcal{I}, \tau)=\{U \backslash E \mid U \in \tau, E \in \mathcal{I}\} .
$$

Note that $\tau^{*}$ is finer than $\tau$. For a subset $A \subset X, \mathrm{Cl}^{*}(A)=A \cup A^{*}$ defined a Kuratowski closure operator for a topology $\tau^{*}$.

Lemma 2.1. [25] Let $(X, \tau)$ be a topological space with ideals $\mathcal{I}$ and $\mathcal{J}$ on $X$. For subsets $A$ and $B$ of $X$, we have the following assertions.

(i) $A \subset B \Rightarrow A^{*} \subset B^{*}$.

(ii) $\mathcal{I} \subset \mathcal{J} \Rightarrow A^{*}(\mathcal{J}) \subset A^{*}(\mathcal{I})$.

(iii) $A^{*}=\mathrm{Cl}\left(A^{*}\right) \subset \mathrm{Cl}(A)\left(A^{*}\right.$ is a closed subset of $\left.\mathrm{Cl}(A)\right)$.

(iv) $\left(A^{*}\right)^{*} \subset A^{*}$. 
(v) $(A \cup B)^{*}=A^{*} \cup B^{*}$.

(vi) $A^{*} \backslash B^{*}=(A \backslash B)^{*} \backslash B^{*} \subset(A \backslash B)^{*}$.

(vii) $U \in \tau \Rightarrow U \cap A^{*}=U \cap(U \cap A)^{*} \subset(U \cap A)^{*}$.

(viii) $B \in \mathcal{I} \Rightarrow(A \cup B)^{*}=A^{*}=(A \backslash B)^{*}$.

Definition 2.2. Let $(X, \tau, \mathcal{I})$ be an ideal topological space. A subset $A$ of $X$ is said to be $\mathcal{I}$-open [26] if $A \subset \operatorname{Int}\left(A^{*}\right)$.

The set of all $\mathcal{I}$-open sets in ideal topological space $(X, \tau, \mathcal{I})$ is denoted by $\mathcal{I} O(X, \tau, \mathcal{I})$ or written simply as $\mathcal{I} O(X)$ when there is no chance for confusion.

Remark 2.3. [26] One can deduce that $\mathcal{I}$-open set $\Rightarrow$ pre-open set, and the converse is not true, in general.

3. Pre(resp. semi and $\alpha$ )-local functions in ideal topological spaces

Definition 3.1. Let $A$ be a subset of an ideal topological space $(X, \tau, \mathcal{I})$. Then the set

$$
A_{p}^{*}(\tau, \mathcal{I})=\left\{x \in X: U \cap A \notin \mathcal{I} \text { for each } U \in \tau^{p}(x)\right\}
$$

is called the pre-local function with respect to $\tau$ and $\mathcal{I}$, where

$$
\tau^{p}(x)=\left\{U \in \tau^{p}: x \in U\right\} .
$$

We will use $A_{p}^{*}$ and/or $A_{p}^{*}(\mathcal{I})$ instead of $A_{p}^{*}(\tau, \mathcal{I})$.

Definition 3.2. Let $(X, \tau, \mathcal{I})$ be an ideal topological space. Then the set

$$
A_{s}^{*}(\tau, \mathcal{I})=\left\{x \in X: U \cap A \notin \mathcal{I} \text { for each } U \in \tau^{s}(x)\right\}
$$

is called the semi-local function with respect to $\tau$ and $\mathcal{I}$, where

$$
\tau^{s}(x)=\left\{U \in \tau^{s}: x \in U\right\} .
$$

We will use $A_{s}^{*}$ and/or $A_{s}^{*}(\mathcal{I})$ instead of $A_{s}^{*}(\tau, \mathcal{I})$.

Definition 3.3. Let $(X, \tau, \mathcal{I})$ be an ideal topological space. Then the set

$$
A_{\alpha}^{*}(\tau, \mathcal{I})=\left\{x \in X: U \cap A \notin \mathcal{I} \text { for each } U \in \tau^{\alpha}(x)\right\}
$$

is called the $\alpha$-local function with respect to $\tau$ and $\mathcal{I}$, where

$$
\tau^{\alpha}(x)=\left\{U \in \tau^{\alpha}: x \in U\right\} .
$$

We will use $A_{\alpha}^{*}$ and/or $A_{\alpha}^{*}(\mathcal{I})$ instead of $A_{\alpha}^{*}(\tau, \mathcal{I})$. 
Example 3.4. Let $X=\{a, b, c, d\}, \tau=\{X, \emptyset,\{c\},\{a, b\},\{a, b, c\}\}$ and $\mathcal{I}=\{\emptyset,\{a\}\}$. Then $(X, \tau, \mathcal{I})$ is an ideal topological space. We know that $\tau^{\alpha}=\tau, \tau^{p}=\{X, \emptyset,\{a\},\{b\},\{c\},\{a, b\},\{a, c\},\{b, c\},\{a, b, c\}$, $\{a, c, d\},\{b, c, d\}\}$ and $\tau^{s}=\{X, \emptyset,\{c\},\{a, b\},\{c, d\},\{a, b, c\},\{a, b, d\}\}$. If $A=\{a, b\}$ then $A_{\alpha}^{*}=\{a, b, d\}, A_{s}^{*}=\{a, b\}$ and $A_{p}^{*}=\{b\}$. If $B=\{a, c\}$, then $B_{\alpha}^{*}=\{c, d\}=B_{p}^{*}$ and $A_{s}^{*}=\{c\}$.

Theorem 3.5. Let $(X, \tau, \mathcal{I})$ be an ideal topological space and let $A$ be a subset of $X$. Then

(i) $A_{p}^{*} \subset A_{\alpha}^{*} \subset A^{*}$.

(ii) $A_{s}^{*} \subset A_{\alpha}^{*} \subset A^{*}$.

(iii) $A_{p}^{*} \subset p \mathrm{Cl}(A), A_{s}^{*} \subset s \mathrm{Cl}(A)$ and $A_{\alpha}^{*} \subset \alpha \mathrm{Cl}(A)$.

Proof. (i) and (ii) are straightforward.

(iii) Let $x \in A_{p}^{*}$. Then $x \in\left\{y \in X: U \cap A \notin \mathcal{I}\right.$ for every $\left.U \in \tau^{p}(y)\right\}$ where $\tau^{p}(y)=\left\{U \in \tau^{p}: y \in U\right\}$. If $x \in A$ then $x \in A \cup D_{p}(A)=p \operatorname{Cl}(A)$ where $D_{p}(A)$ is the pre-derived set of $A$. If $x \notin A$ then $U \cap A \notin \mathcal{I}$ because $x \in A_{p}^{*}$ for every $U \in \tau^{p}(x)$. Since $x \notin A,(U \backslash\{x\}) \cap A \notin \mathcal{I}$. It follows that $(U \backslash\{x\}) \cap A \neq \emptyset$ because $\{\emptyset\} \subset \mathcal{I}$ so that $x \in D_{p}(A) \subset$ $A \cup D_{p}(A)=p \operatorname{Cl}(A)$. Hence $A_{p}^{*} \subset p \operatorname{Cl}(A)$. By the similar way, we can obtain the other results.

Theorem 3.6. Let $(X, \tau, \mathcal{I})$ be an ideal topological space and let $A$ be a subset of $X$. Then

(i) If $\mathcal{I}=\{\emptyset\}$, then $A_{p}^{*}=p \mathrm{Cl}(A), A_{s}^{*}=s \mathrm{Cl}(A)$ and $A_{\alpha}^{*}=\alpha \mathrm{Cl}(A)$.

(ii) If $\mathcal{I}=\mathcal{P}(X)$, then $A_{p}^{*}=A_{s}^{*}=A_{\alpha}^{*}=\emptyset$.

Proof. (i) Let $\mathcal{I}=\{\emptyset\}$. We know that $p \operatorname{Cl}(A)=A \cup D_{p}(A)$ where $D_{p}(A)$ is a pre-derived set of $A$. Let $x \in A \cup D_{p}(A)$ and let $G_{x} \in \tau^{p}$ containing $x$. Then $x \in A$ or $x \in D_{p}(A)$. If $x \in A$ then $x \in G_{x} \cap A$, and so $G_{x} \cap A \neq \emptyset$. If $x \in D_{p}(A)$ then $\emptyset \neq\left(G_{x} \backslash\{x\}\right) \cap A \subset G_{x} \cap A$ and thus $G_{x} \cap A \neq \emptyset$. Hence $p \operatorname{Cl}(A)=A \cup D_{p}(A) \subset A_{p}^{*}$. We know from Theorem 3.5 that $A_{p}^{*} \subset p \operatorname{Cl}(A)$. Therefore $A_{p}^{*}=p \operatorname{Cl}(A)$. Similarly, we have $A_{s}^{*}=s \mathrm{Cl}(A)$ and $A_{\alpha}^{*}=\alpha \mathrm{Cl}(A)$.

(ii) Straightforward.

Lemma 3.7. [19] Let $(X, \tau)$ be a topological space and let $A, B$ be subsets of $X$. If $A \in \tau^{\alpha}$ and $B \in \tau^{p}$ then $A \cap B \in \tau^{p}$.

Lemma 3.8. Let $(X, \tau)$ be a topological space with ideals $\mathcal{I}$ and $\mathcal{J}$ on $X$, and let $A, B$ be subsets of $X$. Then

(i) $A \subset B \Rightarrow A_{p}^{*} \subset B_{p}^{*}$.

(ii) $\mathcal{I} \subset \mathcal{J} \Rightarrow A_{p}^{*}(\mathcal{J}) \subset A_{p}^{*}(\mathcal{I})$. 
(iii) $A_{p}^{*}=p \operatorname{Cl}\left(A_{p}^{*}\right) \subset p \mathrm{Cl}(A)\left(A_{p}^{*}\right.$ is a pre-closed subset of $\left.p \mathrm{Cl}(A)\right)$.

(iv) $\left(A_{p}^{*}\right)_{p}^{*} \subset A_{p}^{*}$.

(v) $B \in \mathcal{I} \Rightarrow B_{p}^{*}=\emptyset$.

(vi) $U \in \tau^{\alpha} \Rightarrow U \cap A_{p}^{*}=U \cap(U \cap A)_{p}^{*} \subset(U \cap A)_{p}^{*}$.

(vii) $B \in \mathcal{I} \Rightarrow(A \cup B)_{p}^{*}=A_{p}^{*}=(A \backslash B)_{p}^{*}$.

(viii) $A_{p}^{*}(\mathcal{I} \cap \mathcal{J}) \supset A_{p}^{*}(\mathcal{I}) \cup A_{p}^{*}(\mathcal{J})$.

Proof. (i) Suppose that $A \subset B$. Let $x \in A_{p}^{*}$. Then $G_{x} \cap A \notin \mathcal{I}$ for every pre-open $G_{x}$ containing $x$. Since $A \subset B$, we have $G_{x} \cap A \subset G_{x} \cap B$. Then $G_{x} \cap B \notin \mathcal{I}$ by the heredity of ideal. Hence $x \in B_{p}^{*}$, and therefore $A_{p}^{*} \subset B_{p}^{*}$.

(ii) Suppose that $\mathcal{I} \subset \mathcal{J}$. Let $x \in A_{p}^{*}(\mathcal{J})$. Then $G_{x} \cap A \notin \mathcal{J}$ for every pre-open $G_{x}$ containing $x$. Since $\mathcal{I} \subset \mathcal{J}$, we get $G_{x} \cap A \notin \mathcal{I}$. Hence $x \in A_{p}^{*}(\mathcal{I})$, and so $A_{p}^{*}(\mathcal{J}) \subset A_{p}^{*}(\mathcal{I})$.

(iii) We will show that $A_{p}^{*}$ is a pre-closed set. Let $x \in\left(A_{p}^{*}\right)^{c}$. Then $x \notin A_{p}^{*}$ and so there exist a pre-open set $U_{x}$ containing $x$ such that $U_{x} \cap A \in \mathcal{I}$. It follows that $y \notin A_{p}^{*}$ for all $y \in U_{x}$ so that $y \in\left(A_{p}^{*}\right)^{c}$. Then $x \in U_{x} \subset\left(A_{p}^{*}\right)^{c}$. Hence $\left(A_{p}^{*}\right)^{c}$ is pre-open. Finally, we know that $A_{p}^{*}$ is pre-closed. Therefore $A_{p}^{*}=p \mathrm{Cl}\left(A_{p}^{*}\right) \subset p \mathrm{Cl}(A)$ by Theorem 3.5.

(iv) By (iii), we obtain $\left(A_{p}^{*}\right)_{p}^{*} \subset p \mathrm{Cl}\left(A_{p}^{*}\right)=A_{p}^{*}$.

(v) Suppose that $B \in \mathcal{I}$. Since $G \cap B \subset B$ for every subset $G$ of $X$, $G \cap B \in \mathcal{I}$ by the heredity of ideal. Hence $B_{p}^{*}=\emptyset$.

(vi) Since $U \cap A \subset A$, (i) implies that $(U \cap A)_{p}^{*} \subset A_{p}^{*}$. Hence $U \cap(U \cap$ $A)_{p}^{*} \subset U \cap A_{p}^{*}$. Let $x \in U \cap A_{p}^{*}$. Then $x \in U$ and $x \in A_{p}^{*}$. It follows that $G \cap A \notin \mathcal{I}$ for every $G \in \tau^{p}(x)=\left\{H \in \tau^{p}: x \in H\right\}$. Since $U$ is an $\alpha$-open set, $U \cap G$ is pre-open containing $x$ by Lemma 3.7 and so $U \cap G \in \tau^{p}(x)$. Thus $(G \cap U) \cap A \notin \mathcal{I}$, and hence $G \cap(U \cap A) \notin \mathcal{I}$. Then $x \in(U \cap A)_{p}^{*}$. Hence $x \in U \cap(U \cap A)_{p}^{*}$. Finally, we have $U \cap A_{p}^{*}=U \cap(U \cap A)_{p}^{*} \subset(U \cap A)_{p}^{*}$.

(vii) By (i), we have that $(A \cup B)_{p}^{*} \supset A_{p}^{*}$. Let $x \in(A \cup B)_{p}^{*}$. Then $U \cap(A \cup B) \notin \mathcal{I}$ for every $U \in \tau^{p}(x)$. This implies that $(U \cap A) \cup(U \cap B) \notin$ $\mathcal{I}$. Since $U \cap B \subset B, U \cap B \in \mathcal{I}$ by the heredity of ideal and so $U \cap A \notin \mathcal{I}$. Hence $x \in A_{p}^{*}$. Therefore $(A \cup B)_{p}^{*} \subset A_{p}^{*}$. Consequently $(A \cup B)_{p}^{*}=A_{p}^{*}$. Since $A_{p}^{*}=((A \backslash B) \cup(A \cap B))_{p}^{*}$, we obtain $A_{p}^{*}=((A \backslash B) \cup(A \cap B))_{p}^{*}=$ $(A \backslash B)_{p}^{*}$ by the first result of (vii).

(viii) It is straightforward by (ii).

In Lemma 3.8, the reverse inclusions of (iii) and (viii) are not valid as seen in the following example. 
Example 3.9. Consider ideal topological spaces $(X, \tau, \mathcal{I}),(X, \tau, \mathcal{J})$ and $(X, \kappa, \mathcal{I})$ where $X=\{a, b, c, d\}, \tau=\{X, \emptyset,\{a, b\},\{a, b, c\}\}$,

$$
\kappa=\{X, \emptyset,\{c\},\{a, b\},\{a, b, c\}\},
$$

$\mathcal{I}=\{\emptyset\{a\}\}$ and $\mathcal{J}=\{\emptyset,\{b\}\}$. If $A=\{a, c\}$ in $(X, \kappa, \mathcal{I})$ then

$$
A_{p}^{*}(\kappa, \mathcal{I})=\{c, d\} \not \supset\{a, c, d\}=p \operatorname{Cl}(A) .
$$

If $A=\{a, b\}$ then $A_{p}^{*}(\tau, \mathcal{I})=\{b\}, A_{p}^{*}(\tau, \mathcal{J})=\{a\}$ and $A_{p}^{*}(\tau, \mathcal{I} \cap \mathcal{J})=$ $\{a, b, c, d\}$. Hence we know that $A_{p}^{*}(\tau, \mathcal{I} \cap \mathcal{J}) \not \subset A_{p}^{*}(\tau, \mathcal{I}) \cup A_{p}^{*}(\tau, \mathcal{J})$.

The following example shows that the identity $A_{p}^{*} \backslash B_{p}^{*}=(A \backslash B)_{p}^{*} \backslash B_{p}^{*}$ and the inclusions $A_{p}^{*} \backslash B_{p}^{*} \subset(A \backslash B)_{p}^{*}$ and $A_{p}^{*} \backslash B_{p}^{*} \supset(A \backslash B)_{p}^{*}$ are not valid.

Example 3.10. Let $X=\{a, b, c, d\}, \tau=\{X, \emptyset,\{a, b\},\{a, b, c\}\}$ and $\mathcal{I}=\{\emptyset\}$. Then $(X, \tau, \mathcal{I})$ is an ideal topological space. Take $A=\{a, b\}$, $B=\{a, d\}$. Then $A_{p}^{*}=\{a, b, c, d\}, B_{p}^{*}=\{a, d\}$ and $(A \backslash B)_{p}^{*}=\{b\}$. Thus $A_{p}^{*} \backslash B_{p}^{*}=\{b, c\} \neq\{b\}=(A \backslash B)_{p}^{*} \backslash B_{p}^{*}$.

If $A=\{a, b\}, B=\{a\}$ then $A_{p}^{*}=\{a, b, c, d\}, B_{p}^{*}=\{a\}$. Hence $A_{p}^{*} \backslash B_{p}^{*}=\{b, c, d\} \not \subset\{b\}=\{b\}_{p}^{*}=(A \backslash B)_{p}^{*}$.

If $A=\{a, b, c, d\}, B=\{a, b, c\}$ then $A_{p}^{*}=\{a, b, c, d\}, B_{p}^{*}=\{a, b, c, d\}$. Hence $A_{p}^{*} \backslash B_{p}^{*}=\emptyset \not \supset\{d\}=\{d\}_{p}^{*}=(A \backslash B)_{p}^{*}$.

The converses of (i), (ii), (vi) and (vii) in Lemma 3.8 may not be true as seen in the the following example.

Example 3.11. Let $X=\{a, b, c, d\}, \tau=\{X, \emptyset,\{c\},\{a, b\},\{a, b, c\}\}$, $\mathcal{I}=\{\emptyset,\{a\}\}$ and $\mathcal{J}=\{\{\emptyset,\{c\}\}$. Then $(X, \tau, \mathcal{I})$ and $(X, \tau, \mathcal{J})$ are ideal topological spaces.

(i) If $A=\{b, c\}$ and $B=\{a, b, d\}$ then $A_{p}^{*}(\mathcal{I})=\{b, c, d\}$ and $B_{p}^{*}(\mathcal{I})=$ $\{b, d\}$. Hence we know that $A_{p}^{*}(\mathcal{I}) \supset B_{p}^{*}(\mathcal{I})$ but $A \not \supset B$.

(ii) If $A=\{b, c\}$ then $A_{p}^{*}(\mathcal{I})=\{b, c, d\}$ and $A_{p}^{*}(\mathcal{J})=\{b\}$. Thus $A_{p}^{*}(\mathcal{J}) \subset A_{p}^{*}(\mathcal{I})$ but $\mathcal{I} \not \subset \mathcal{J}$.

(vi) If $A=\{a, b, c\}$ and $U=\{a, c, d\}$ then $(U \cap A)_{p}^{*}(\mathcal{I})=U \cap(U \cap$ $A)_{p}^{*}(\mathcal{I})=\{c, d\}$ and $U \cap A_{p}^{*}(\mathcal{I})=\{c, d\}$. Thus

$$
U \cap A_{p}^{*}(\mathcal{I})=U \cap(U \cap A)_{p}^{*}(\mathcal{I}) \subset(U \cap A)_{p}^{*}(\mathcal{I})
$$

but $U \notin \tau^{\alpha}$.

(vii) If $A=\{b, c, d\}$ and $B=\{d\}$ then $(A \cup B)_{p}^{*}(\mathcal{I})=A_{p}^{*}(\mathcal{I})=\{b, c, d\}$ and $(A \backslash B)_{p}^{*}(\mathcal{I})=\{b, c, d\}$. Thus $(A \cup B)_{p}^{*}(\mathcal{I})=A_{p}^{*}(\mathcal{I})=(A \backslash B)_{p}^{*}(\mathcal{I})$ but $B \notin \mathcal{I}$. 
Lemma 3.12. For a class $\left\{U_{i} \mid i \in \Lambda\right\}$ of subsets of an ideal topological space $(X, \tau, \mathcal{I})$, we have

(i) $\bigcup_{i \in \Lambda}\left(\left(U_{i}\right)_{p}^{*}\right) \subset\left(\bigcup_{i \in \Lambda}\left(U_{i}\right)\right)_{p}^{*}$.

(ii) $\bigcap_{i \in \Lambda}^{i \in \Lambda}\left(\left(U_{i}\right)_{p}^{*}\right) \supset\left(\bigcap_{i \in \Lambda}\left(U_{i}\right)\right)_{p}^{*}$.

Proof. Straightforward by Lemma 3.8.

The reverse inclusion of (i) in Lemma 3.12 is not valid as seen in the following example.

Example 3.13. Let $X=\{a, b, c, d\}, \tau=\{X, \emptyset,\{a, b\},\{a, b, c\}\}$ and $\mathcal{I}=\{\emptyset\}$. Then $(X, \tau, \mathcal{I})$ is an ideal topological space. We note that $\tau^{p}=$ $\{X, \emptyset,\{a\},\{b\},\{a, b\},\{a, c\},\{a, d\},\{b, c\},\{b, d\},\{a, b, c\},\{a, b, d\},\{a, c, d\}$, $\{b, c, d\}\}$. If $A=\{a\}$ and $B=\{b\}$ then $A_{p}^{*}=\{a\}, B_{p}^{*}=\{b\}$ and $(A \cup B)_{p}^{*}=\{a, b, c, d\}$. Hence $A_{p}^{*} \cup B_{p}^{*} \not \supset(A \cup B)_{p}^{*}$.

Lemma 3.14. For any subset $A$ of an ideal topological space $(X, \tau, \mathcal{I})$, if $A \subset A_{p}^{*}$ then $A_{p}^{*}=p \mathrm{Cl}\left(A_{p}^{*}\right)=p \mathrm{Cl}(A)$.

Proof. Straightforward.

Theorem 3.15. Let $(X, \tau, \mathcal{I})$ be an ideal topological space. Then

$$
A_{p}^{*} \supset A \backslash \cup\{U \subset X: U \in \mathcal{I}\}
$$

for all $A \subset X$.

Proof. Put $B:=\cup\{U \subset X: U \in \mathcal{I}\}$ and let $x \in A \backslash B$. Then $x \in A$ and $x \notin B$. This implies that $x \notin U$ for all $U \in \mathcal{I}$ so that $\{x\}=\{x\} \cap A \notin \mathcal{I}$ because $x \in A$. For every $G \in \tau^{p}(x)$, we have $\{x\} \cap A \subset G \cap A \notin \mathcal{I}$ by the heredity of ideal. Hence $x \in A_{p}^{*}$. This completes the proof.

The reverse inclusion of Theorem 3.15 is not valid as seen in the following example.

Example 3.16. Consider an ideal topological space $(X, \tau, \mathcal{I})$ where $X=\{a, b, c, d\}, \tau=\{X, \emptyset,\{c\},\{a, b\},\{a, b, c\}\}$ and $\mathcal{I}=\{\emptyset,\{a\}\}$. Then $B:=\cup\{U \subset X: U \in \mathcal{I}\}=\{a\}$. If $A=\{a, c\}$ then $A_{p}^{*}=\{c, d\}$. Hence $A_{p}^{*}=\{c, d\} \not \subset\{c\}=A \backslash B$.

Theorem 3.17. Let $(X, \tau, \mathcal{I})$ be an ideal topological space. Put

$$
B:=\cup\{U \subset X: U \in \mathcal{I}\} .
$$

If $B \in \mathcal{I}$ then $\left(A_{p}^{*}\right)_{p}^{*}=A_{p}^{*}$ for all $A \subset X$. 
Proof. Let $A$ be a subset of $X$. Then $\left(A_{p}^{*}\right)_{p}^{*} \subset A_{p}^{*}$ by Lemma 3.8(iv). Furthermore $A_{p}^{*} \supset A \backslash B$ by Theorem 3.15. It follows from Lemma 3.8(i) that $\left(A_{p}^{*}\right)_{p}^{*} \supset(A \backslash B)_{p}^{*}$. Since $B \in \mathcal{I}$, Lemma 3.8(vii) implies that $\left(A_{p}^{*}\right)_{p}^{*} \supset(A \backslash B)_{p}^{*}=A_{p}^{*}$. Therefore $\left(A_{p}^{*}\right)_{p}^{*}=A_{p}^{*}$.

Theorem 3.18. Let $(X, \tau, \mathcal{I})$ be an ideal topological space in which the cardinality of $\mathcal{I}$ is finite. Then $\left(A_{p}^{*}\right)_{p}^{*}=A_{p}^{*}$ for all $A \subset X$.

Proof. Let $B:=\cup\{U \subset X: U \in \mathcal{I}\}$. Since the cardinality of $\mathcal{I}$ is finite, $B \in \mathcal{I}$ by the finite additivity of ideal. Therefore $\left(A_{p}^{*}\right)_{p}^{*}=A_{p}^{*}$ for all $A \subset X$ by Theorem 3.17.

Corollary 3.19. Let $(X, \tau, \mathcal{I})$ be an ideal topological space in which the cardinality of $X$ is finite. Then $\left(A_{p}^{*}\right)_{p}^{*}=A_{p}^{*}$ for all $A \subset X$.

Proof. Straightforward.

Theorem 3.20. Let $(X, \tau, \mathcal{I})$ be an ideal topological space in which $\tau^{p}=\mathcal{P}(X)$. Then

$$
A_{p}^{*}=A \backslash \cup\{U \subset X: U \in \mathcal{I}\}
$$

for all $A \subset X$.

Proof. Put $B:=\cup\{U \subset X: U \in \mathcal{I}\}$ and let $x \in A_{p}^{*}$. Then $\{x\} \cap A \notin$ $\mathcal{I}$ because $\{x\} \in \tau^{p}=\mathcal{P}(X)$. Since ideal $\mathcal{I}$ always contain $\emptyset,\{x\} \cap A \neq \emptyset$ and so $x \in A$. It follows that $\{x\}=\{x\} \cap A \notin \mathcal{I}$ so that $x \notin U$ for all $U \in \mathcal{I}$. Hence $x \notin B$, and therefore $x \in A \backslash B$. Hence $A_{p}^{*} \subset A \backslash B$. The reverse inclusion is obvious by Theorem 3.15.

Corollary 3.21. Let $(X, \tau, \mathcal{I})$ be an ideal topological space in which every member of $\tau$ is clopen. Then

$$
A_{p}^{*}=A \backslash \cup\{U \subset X: U \in \mathcal{I}\}
$$

for all $A \subset X$. or all $A \subset X$.

Proof. Put $B:=\cup\{U \subset X: U \in \mathcal{I}\}$ and let $A \in \mathcal{P}(X)$. Then $A \subset \mathrm{Cl}(A)=\operatorname{Int}(\mathrm{Cl}(A))$ because every member of $\tau$ is clopen. Hence $A \in \tau^{p}$, which means that $\mathcal{P}(X) \subset \tau^{p}$ so that $\mathcal{P}(X)=\tau^{p}$. By Theorem $3.20, A_{p}^{*}=A \backslash B$.

Lemma 3.22. Let $(X, \tau)$ be a topological space. Then the set

$$
\{U \subset X: U \cap G \neq \emptyset \text { for all } G \in \tau \backslash\{\emptyset\}\}
$$

is contained in $\tau^{p}$. 
Proof. Let $H \in\{U \subset X: U \cap G \neq \emptyset$ for all $G \in \tau \backslash\{\emptyset\}\}$. For any $a \in X$, if $a \in H$ then clearly $a \in \mathrm{Cl}(H)$. If $a \notin H$ then $H \cap$ $(F \backslash\{a\})=H \cap F \neq \emptyset$ for any $F \in \tau \backslash\{\emptyset\}$ containing $a$ because $H \in\{U \subset X: U \cap G \neq \emptyset$ for all $G \in \tau \backslash\{\emptyset\}\}$. Thus $a \in D(H) \subset \mathrm{Cl}(H)$ where $D(H)$ is the derived set of $H$. It follows that $\mathrm{Cl}(H)=X$ so that $H \subset X=\operatorname{Int}(\mathrm{Cl}(H))$. Hence $H \in \tau^{p}$. Therefore the result is valid.

Lemma 3.23. Let $(X, \tau)$ be a topological space. If $\tau$ is a chain under the set inclusion then

$$
\tau^{p}=\{U \subset X: U \cap G \neq \emptyset \text { for all } G \in \tau \backslash\{\emptyset\}\} \cup\{\emptyset\} .
$$

Proof. Since $\emptyset \in \tau^{p}, \tau^{p} \supset\{U \subset X: U \cap G \neq \emptyset$ for all $G \in \tau \backslash\{\emptyset\}\} \cup$ $\{\emptyset\}$ by Lemma 3.22.

Conversely, let $H \in \tau^{p}$. Then $H \subseteq \operatorname{Int}(\mathrm{Cl}(H))$. If $H=\emptyset$ then clearly $H \in\{U \subset X: U \cap G \neq \emptyset$ for all $G \in \tau \backslash\{\emptyset\}\} \cup\{\emptyset\}$. Suppose that $H \neq \emptyset$. If there exist $F \in \tau \backslash\{\emptyset\}$ such that $H \cap F=\emptyset$, then $H \subset F^{c}$ and so $\operatorname{Int}(\mathrm{Cl}(H)) \subset \operatorname{Int}\left(\mathrm{Cl}\left(F^{c}\right)\right)=\operatorname{Int}\left(F^{c}\right)$. Since $\tau$ is a chain under the set inclusion, $I \cap F \neq \emptyset$ for all $I \in \tau \backslash\{\emptyset\}$. It follows that $I \not \subset F^{c}$ so that $\operatorname{Int}\left(F^{c}\right)=\emptyset$. Thus $H \subseteq \operatorname{Int}(\mathrm{Cl}(H)) \subset \operatorname{Int}\left(\mathrm{Cl}\left(F^{c}\right)\right)=\operatorname{Int}\left(F^{c}\right)=\emptyset$. This is a contradiction. Hence $H \cap F=\emptyset$ for all $F \in \tau \backslash\{\emptyset\}$. Therefore $\tau^{p}=\{U \subset X: U \cap G \neq \emptyset$ for all $G \in \tau \backslash\{\emptyset\}\} \cup\{\emptyset\}$.

Theorem 3.24. Let $(X, \tau, \mathcal{I})$ be an ideal topological space. If $\tau$ is a chain under the set inclusion, then either $G_{p}^{*}=X$ or $G_{p}^{*} \subset G$ for $G \subset X$.

Proof. Let $G$ be a subset of $X$. Suppose that $G_{p}^{*} \neq X$. We will show that $G \subseteq G_{p}^{*}$. Let $x \notin G$. Since $G_{p}^{*} \neq X$, there exists $a \in X$ such that $a \notin G_{p}^{*}$. This implies that there exists $H \in \tau^{p}(a)$ such that $H \cap G \in \mathcal{I}$. Since $\tau$ is a chain under the set inclusion, $H \cap F \neq \emptyset$ for each $F \in \tau \backslash\{\emptyset\}$ by Lemma 3.23. Then $(H \cup\{x\}) \cap F \neq \emptyset$ for each $F \in \tau \backslash\{\emptyset\}$ and so, $x \in H \cup\{x\} \in \tau^{p}$ by Lemma 3.23. Furthermore $(H \cup\{x\}) \cap G=H \cap G \in \mathcal{I}$. Hence $x \notin G_{p}^{*}$. Therefore we obtain the desired result $G_{p}^{*} \subseteq G$.

Theorem 3.25. Let $(X, \tau, \mathcal{I})$ be an ideal topological space. Assume that $\tau$ is a chain under the set inclusion in which there exists the smallest element $A$ of $(\tau \backslash\{\emptyset\}, \subset)$. If $A \cap B=\emptyset$ for every $B \in \mathcal{I}$, then $G_{p}^{*}=X$ for $G \subset X$ containing $A$.

Proof. Let $A \subset G$. Assume that there exists $x \in X$ such that $x \notin G_{p}^{*}$. Then there exists $H \in \tau^{p}(x)=\left\{U \in \tau^{p}: x \in U\right\}$ such that $H \cap G \in \mathcal{I}$. Since $H \in \tau^{p}(x), H \cap A \neq \emptyset$ by Lemma 3.23. Since $H \cap G \in \mathcal{I}$, $A \cap(H \cap G)=\emptyset$ by the hypothesis. It follows that $A \cap H=A \cap(H \cap G)=\emptyset$ because $A \subset G$. This is a contradiction. Therefore $x \in G_{p}^{*}$. 


\section{4. $P$-I-open sets and $P$-I-closed sets}

Definition 4.1. Let $(X, \tau, \mathcal{I})$ be an ideal topological space. A subset $A$ of $X$ is said to be $P$ - $\mathcal{I}$-open if $A \subset p \operatorname{Int}\left(A_{p}^{*}\right)$. A subset $B$ of $X$ is said to be $P$ - $\mathcal{I}$-closed if the complement of $B$ is $P$ - $\mathcal{I}$-open.

The set of all $P$ - $\mathcal{I}$-open sets in $(X, \tau, \mathcal{I})$ is denoted by $P \mathcal{I} O(X, \tau, \mathcal{I})$. Simply $P \mathcal{I} O(X, \tau, \mathcal{I})$ is written as $P \mathcal{I} O(X)$ or $P \mathcal{I} O(X, \tau)$ when there is no chance for confusion.

Lemma 4.2. [24] Let $S$ be a set in $(X, \tau)$. Then

(i) $p \mathrm{Cl}(S)=S \cup \mathrm{Cl}(\operatorname{Int}(S))$, $p \operatorname{Int}(S)=S \cap \operatorname{Int}(\mathrm{Cl}(S))$.

(ii) $\operatorname{Int}(s \mathrm{Cl}(S))=p \operatorname{Int}(\mathrm{Cl}(S))=p \operatorname{Int}(s \mathrm{Cl}(S))=s \mathrm{Cl}(p \operatorname{Int}(S))=\operatorname{Int}(\mathrm{Cl}(S))$.

(iii) $\operatorname{Int}(p \mathrm{Cl}(S))=s \mathrm{Cl}(\operatorname{Int}(S))=\operatorname{Int}(\mathrm{Cl}(\operatorname{Int}((S)))$.

Proposition 4.3. For any set $A$ in $(X, \tau, \mathcal{I})$, we have

$$
p \operatorname{Int}\left(A_{p}^{*}\right) \subseteq \operatorname{Int}\left(A^{*}\right) .
$$

Proof. We have

$$
\begin{aligned}
p \operatorname{Int}\left(A_{p}^{*}\right) & =A_{p}^{*} \cap \operatorname{Int}\left(\operatorname{Cl}\left(A_{p}^{*}\right)\right) \quad(\text { by Lemma } 4.2) \\
& \subset \operatorname{Int}\left(\operatorname{Cl}\left(A_{p}^{*}\right)\right) \\
& \subset \operatorname{Int}\left(\mathrm{Cl}\left(A^{*}\right)\right) \quad(\text { by Theorem } 3.5) \\
& =\operatorname{Int}\left(A^{*}\right) \quad(\text { by Lemma } 2.1)
\end{aligned}
$$

This completes the proof.

Theorem 4.4. Let $A \in P \mathcal{I} O(X)$. Then $A$ is $\mathcal{I}$-open.

Proof. It is straightforward by Proposition 4.3.

The converse of Theorem 4.4 may not be true as seen in the following example.

Example 4.5. Let $X=\{a, b, c, d\}, \tau=\{X, \emptyset,\{c\},\{a, b\},\{a, b, c\}\}$ and $\mathcal{I}=\{\emptyset,\{a\}\}$. Then $(X, \tau, \mathcal{I})$ be an ideal topological space. We have that

$\tau^{p}=\{X, \emptyset,\{a\},\{b\},\{c\},\{a, b\},\{a, c\},\{b, c\},\{a, b, c\},\{a, c, d\},\{b, c, d\}\}$. Since $\operatorname{Int}\left(\{a, b\}^{*}\right)=\operatorname{Int}(\{a, b, d\})=\{a, b\}$, we have $\{a, b\} \in \mathcal{I} O(X)$. But $\{a, b\} \notin P \mathcal{I} O(X)$ because $p \operatorname{Int}\left(\{a, b\}_{p}^{*}\right)=p \operatorname{Int}(\{b\})=\{b\}$.

Lemma 4.6. [27] Let $A$ be a subset of a topological space $(X, \tau)$. Then the following assertions are satisfied.

(i) $(p \operatorname{Int}(A))^{c}=p \operatorname{Cl}\left(A^{c}\right)$. 
(ii) $(p \operatorname{Cl}(A))^{c}=p \operatorname{Int}\left(A^{c}\right)$.

Theorem 4.7. If $A$ is $P$-I-closed in an ideal topological space $(X, \tau, \mathcal{I})$, then $A \supset(p \operatorname{Int}(A))_{p}{ }^{*}$.

Proof. Since $A$ is $P$ - $\mathcal{I}$-closed, $A^{c}$ is $P$ - $\mathcal{I}$-open. Thus

$$
\begin{aligned}
A^{c} & \subset p \operatorname{Int}\left(\left(A^{c}\right)_{p}^{*}\right) \\
& \subset p \operatorname{Int}\left(p \operatorname{Cl}\left(A^{c}\right)\right) \\
& =(p \operatorname{Cl}(p \operatorname{Int}(A)))^{c} \quad(\text { by Lemma 4.6 }) .
\end{aligned}
$$

Hence $A \supset p \operatorname{Cl}(p \operatorname{Int}(A)) \supset(p \operatorname{Int}(A))_{p}^{*}$ by Theorem 3.5.

Theorem 4.8. Let $(X, \tau, \mathcal{I})$ be an ideal topological space. Let $A$ be a subset of $X$ such that $\left((p \operatorname{Int}(A))_{p}^{*}\right)^{c}=p \operatorname{Int}\left(\left(A^{c}\right)_{p}^{*}\right)$. Then $A$ is $P$-I -closed if and only if $A \supset(p \operatorname{Int}(A))_{p}^{*}$.

Proof. Let $A$ be a subset of $X$ such that $\left((p \operatorname{Int}(A))_{p}^{*}\right)^{c}=p \operatorname{Int}\left(\left(A^{c}\right)_{p}^{*}\right)$. Suppose that $A$ is $P$-I-closed. Then $A \supset(p \operatorname{Int}(A))_{p}^{*}$ by Theorem 4.7.

Conversely, suppose that $A \supset(p \operatorname{Int}(A))_{p}^{*}$. Then $A^{c} \subset\left((p \operatorname{Int}(A))_{p}^{*}\right)^{c}=$ $p \operatorname{Int}\left(\left(A^{c}\right)_{p}^{*}\right)$. Hence $A$ is $P$ - $\mathcal{I}$-closed.

Theorem 4.9. For any $P$-I-open set $A$ in an ideal topological space $(X, \tau, \mathcal{I})$, we have $A_{p}^{*}=\left(p \operatorname{Int}\left(A_{p}^{*}\right)\right)_{p}^{*}$.

Proof. Let $A$ be a $P$ - $\mathcal{I}$-open set. Then $A \subset p \operatorname{Int}\left(A_{p}^{*}\right)$. By Lemma 3.8 , we know that $A_{p}^{*} \subset\left(p \operatorname{Int}\left(A_{p}^{*}\right)\right)_{p}^{*}$. In general, since $A_{p}^{*} \supset p \operatorname{Int}\left(A_{p}^{*}\right)$, it follows from Lemma 3.8 that $A_{p}^{*} \supset\left(A_{p}^{*}\right)_{p}^{*} \supset\left(p \operatorname{Int}\left(A_{p}^{*}\right)\right)_{p}^{*}$. Therefor we obtain the desired result.

Theorem 4.10. Let $\left\{U_{i} \in P \mathcal{I} O(X): i \in \Lambda\right\}$ be a class of $P$-I -open sets in an ideal topological space $(X, \tau, \mathcal{I})$. Then $\bigcup_{i \in \Lambda}\left\{U_{i} \in P \mathcal{I} O(X): i \in\right.$ $\Lambda$ \} is $P$ - $\mathcal{I}$-open.

Proof. Note that $U_{i} \subset p \operatorname{Int}\left(\left(U_{i}\right)_{p}^{*}\right)$ for every $i \in \Lambda$. It follows that

$$
\begin{aligned}
\bigcup_{i \in \Lambda} U_{i} & \subset \bigcup_{i \in \Lambda} p \operatorname{Int}\left(\left(U_{i}\right)_{p}^{*}\right) \\
& \subset p \operatorname{Int}\left(\bigcup_{i \in \Lambda}\left(U_{i}\right)_{p}^{*}\right) \\
& \subset p \operatorname{Int}\left(\left(\bigcup_{i \in \Lambda}\left(U_{i}\right)\right)_{p}^{*}\right) \quad(\text { by Lemma 3.12) }
\end{aligned}
$$

so that $\bigcup_{i \in \Lambda}\left\{U_{i} \in P \mathcal{I} O(X): i \in \Lambda\right\}$ is $P$ - $\mathcal{I}$-open. 
The intersection of two $P$ - $\mathcal{I}$-open sets need not be $P$ - $\mathcal{I}$-open as seen in following example.

Example 4.11. Consider an ideal topological space $(X, \tau, \mathcal{I})$ where $X=\{a, b, c, d\}, \tau=\{X, \emptyset,\{a, b\},\{a, b, c\}\} \mathcal{I}=\{\emptyset\}$. Then $\tau^{p}=\{X, \emptyset$, $\{a\},\{b\},\{a, b\},\{a, c\},\{a, d\},\{b, c\},\{b, d\},\{a, b, c\},\{a, b, d\},\{a, c, d\}$, $\{b, c, d\}\}$. Note that $\{a, c\},\{b, c, d\} \in P \mathcal{I} O(X)$, but $\{a, c\} \cap\{b, c, d\}=$ $\{c\} \notin P \mathcal{I} O(X)$.

Theorem 4.12. Let $(X, \tau, \mathcal{I})$ be an ideal topological space. If $W$ is a $P$-I- -open and $\tau^{p}$ is a topology, then $p \mathrm{Cl}(V) \cap W \subset(V \cap W)_{p}^{*}$ for every $V \in \tau^{\alpha}$.

Proof. Suppose that $W$ is a $P$ - $\mathcal{I}$-open and $\tau^{p}$ is a topology. Let $V \in \tau^{\alpha}$. Then $V \subset \operatorname{Int}(\mathrm{Cl}(\operatorname{Int}(V)))$. It follows that

$$
\begin{aligned}
p \mathrm{Cl}(V) \cap W & \subset p \mathrm{Cl}(V) \cap p \operatorname{Int}\left(W_{p}^{*}\right) \\
& \subset p \mathrm{Cl}\left(V \cap p \operatorname{Int}\left(W_{p}^{*}\right)\right) \quad(\text { by }[27, \text { Theorem 4.17]) } \\
& \subset p \operatorname{Cl}\left(V \cap W_{p}^{*}\right) \\
& \subset p \operatorname{Cl}(V \cap W)_{p}^{*} \quad(\text { by Lemma 3.8) } \\
& =(V \cap W)_{p}^{*} .
\end{aligned}
$$

This completes the proof.

Lemma 4.13. Let $(X, \tau)$ be a topological space. Let $A$ be a subset of $X$ and let $B \in \tau^{\alpha}$. Then $p \operatorname{Int}(A) \cap B=p \operatorname{Int}(A \cap B)$.

Proof. Since $p \operatorname{Int}(A \cap B) \subset p \operatorname{Int}(A)$ and $p \operatorname{Int}(A \cap B) \subset B$, we have

$$
p \operatorname{Int}(A \cap B) \subset p \operatorname{Int}(A) \cap B .
$$

Conversely, let $x \in p \operatorname{Int}(A) \cap B$. Then $x \in p \operatorname{Int}(A)$ and $x \in B$. Since $x \in p \operatorname{Int}(A)$, there exists pre-open set $G_{x}$ containing $x$ such that $G_{x} \subset A$. Since $x \in B$ and $B \in \tau^{\alpha}, G_{x} \cap B \in \tau^{p}$ by Lemma 3.7 and $x \in G_{x} \cap B \subset$ $A \cap B$. Hence $x \in p \operatorname{Int}(A \cap B)$. Therefore $p \operatorname{Int}(A) \cap B=p \operatorname{Int}(A \cap B)$.

Theorem 4.14. Let $(X, \tau, \mathcal{I})$ be an ideal topological space and let $A, B$ be subsets of $X$. If $A \in P \mathcal{I} O(X, \tau)$ and $B \in \tau^{\alpha}$ then $A \cap B \subset$ $p \operatorname{Int}\left(B \cap(A \cap B)_{p}^{*}\right)$.

Proof. Let $A \in P \mathcal{I} O(X, \tau)$ and $B \in \tau^{\alpha}$. Then

$$
\begin{aligned}
A \cap B & \subset p \operatorname{Int}\left(A_{p}^{*}\right) \cap B \\
& =p \operatorname{Int}\left(A_{p}^{*} \cap B\right) \quad(\text { by Lemma } 4.13) \\
& =p \operatorname{Int}\left(B \cap(A \cap B)_{p}^{*}\right) . \quad(\text { by Lemma } 3.8)
\end{aligned}
$$

This completes the proof. 
If $A$ is not $P$ - $\mathcal{I}$-open but $\mathcal{I}$-open in an ideal topological space $(X, \tau, \mathcal{I})$ then is the above theorem valid? The answer is negative as seen in the following example.

Example 4.15. Consider the ideal topological space $(X, \tau, \mathcal{I})$ which is presented in Example 3.4. Let $A=\{a, b\}$ and $B=\{a, b, c\}$. Then $A$ is $\mathcal{I}$-open which is not $P$ - $\mathcal{I}$-open, and $B \in \tau^{\alpha}$. But $A \cap B=\{a, b\} \not \subset$ $\{b\}=p \operatorname{Int}\left(B \cap(A \cap B)_{p}^{*}\right)$.

Theorem 4.16. Let $(X, \tau, \mathcal{I})$ be an ideal topological space and let $A, B$ be subsets of $X$. If $A \in P \mathcal{I} O(X, \tau)$ and $B \in \tau^{\alpha}$ then $A \cap B \in$ $P \mathcal{I} O(X, \tau)$.

Proof. Let $A \in P \mathcal{I} O(X, \tau)$ and $B \in \tau^{\alpha}$. Then

$$
\begin{aligned}
A \cap B & \subset p \operatorname{Int}\left(A_{p}^{*}\right) \cap B \\
& =p \operatorname{Int}\left(A_{p}^{*} \cap B\right) \quad(\text { by Lemma } 4.13) \\
& \subset p \operatorname{Int}\left((A \cap B)_{p}^{*}\right), \quad(\text { by Lemma } 3.8)
\end{aligned}
$$

and therefore $A \cap B \in P \mathcal{I} O(X, \tau)$.

If $A \in P \mathcal{I} O(X, \tau)$ and $B \in \tau^{p}$ then is $A \cap B P$ - $\mathcal{I}$-open ? The answer is negative as seen in the following example.

Example 4.17. Consider an ideal topological space $(X, \tau, \mathcal{I})$ where $X=\{a, b, c, d\} \tau=\{X, \emptyset,\{c\},\{a, b\},\{a, b, c\}\}$ and $\mathcal{I}=\{\emptyset,\{a\}\}$. Then $\tau^{p}=\{\emptyset,\{a\},\{b\},\{c\},\{a, b\},\{a, c\},\{b, c\},\{a, b, c\},\{a, c, d\},\{b, c, d\}, X\}$. Let $A=\{b, c, d\}$ and $B=\{a, c, d\}$. Then $A \in P \mathcal{I} O(X, \tau)$ and $B \in \tau^{p}$. Since $(A \cap B)_{p}^{*}=\{c, d\}, p \operatorname{Int}\left((A \cap B)_{p}^{*}\right)=p \operatorname{Int}(\{c, d\})=\{c\}$. Hence $A \cap B=\{c, d\} \not \subset p \operatorname{Int}\left((A \cap B)_{p}^{*}\right)=\{c\}$. Therefore $A \cap B \notin P \mathcal{I} O(X, \tau)$.

If $A \in \mathcal{I} O(X, \tau)$ and $B \in \tau^{\alpha}$ then is $A \cap B P$-I -open ? The answer is negative as seen in the following example.

Example 4.18. Consider the ideal topological space $(X, \tau, \mathcal{I})$ in Example 4.17. Take $A=\{a, b, c\} \in \mathcal{I} O(X) \backslash P \mathcal{I} O(X)$ and $B=\{a, b\} \in$ $\tau \subset \tau^{\alpha}$. Then $A \cap B=\{a, b\} \not \subset\{b\}=p \operatorname{Int}\left(\{a, b\}_{p}^{*}\right)=p \operatorname{Int}\left((A \cap B)_{p}^{*}\right)$. Hence $A \cap B$ is not $P$ - $\mathcal{I}$-open.

Corollary 4.19. The union of $P$-I-closed set and $\alpha$-closed set is $P$-I-closed set.

Proof. Let $A$ be a $P$-I-closed set and $B$ be an $\alpha$-closed set. Then $A^{c}$ is a $P$ - $\mathcal{I}$-open set and $B^{c}$ is an $\alpha$-open set. By Theorem 4.16, $(A \cup B)^{c}=$ $A^{c} \cap B^{c} \in P \mathcal{I} O(X, \tau)$. Hence $A \cup B$ is $P$ - $\mathcal{I}$-closed. 
Theorem 4.20. Let $(X, \tau, \mathcal{I})$ be an ideal topological space and let $A$ be a subset of $X$. If $A$ is $P$-I-open and semi-closed, then $A=p \operatorname{Int}\left(A_{p}^{*}\right)$.

Proof. Since $A$ is $P$ - $\mathcal{I}$-open, $A \subset p \operatorname{Int}\left(A_{p}^{*}\right)$. Since $A$ is semi-closed, $A^{c} \subset \mathrm{Cl}\left(\operatorname{Int}\left(A^{c}\right)\right)$. Thus

$$
\begin{aligned}
A^{c} & \subset \operatorname{Cl}\left(\operatorname{Int}\left(A^{c}\right)\right) \\
& =p \operatorname{Cl}\left(\operatorname{Int}\left(A^{c}\right)\right) \quad(\text { by Lemma 4.2) } \\
& \subset p \operatorname{Cl}\left(p \operatorname{Int}\left(A^{c}\right)\right. \\
& =(p \operatorname{Int}(p \operatorname{Cl}(A)))^{c} . \quad(\text { by Lemma 4.6 }) .
\end{aligned}
$$

Hence $A \supset p \operatorname{Int}(p \operatorname{Cl}(A)) \supset p \operatorname{Int}\left(A_{p}^{*}\right)$, and therefore $A=p \operatorname{Int}\left(A_{p}^{*}\right)$.

If $A \in \mathcal{I} O(X, \tau)$ is semi-closed then is $A=p \operatorname{Int}\left(A_{p}^{*}\right)$ valid? The answer is negative as seen in the following example.

Example 4.21. Consider the ideal topological space $(X, \tau, \mathcal{I})$ in Example 4.17. Take $A=\{a, b\}$. Then $A$ is both semi-closed and $\mathcal{I}$-open but not $P$ - $\mathcal{I}$-open. We know that $p \operatorname{Int}\left(A_{p}^{*}\right)=\{b\} \neq A$.

Definition 4.22. A set $S$ in $(X, \tau)$ is called b-open [24] if

$$
S \subseteq \mathrm{Cl}(\operatorname{Int}(S)) \cup \operatorname{Int}(\mathrm{Cl}(S)) .
$$

Theorem 4.23. If $A \subset W \subset p \operatorname{Cl}(A)$ and $A \in \mathcal{I} O(X, \tau)$, then $W$ is b-open.

Proof. Let $A \subset W \subset p \mathrm{Cl}(A)$ and $A \in \mathcal{I} O(X, \tau)$. Then $A \subset \operatorname{Int}\left(A^{*}\right)$ and $\mathrm{Cl}(W)=\mathrm{Cl}(A)$. This implies that

$$
\begin{aligned}
W & \subset p \mathrm{Cl}(A) \\
& =A \cup \mathrm{Cl}(\operatorname{Int}(A)) \quad(\text { by Lemma } 4.2) \\
& \subset \operatorname{Int}\left(A^{*}\right) \cup \mathrm{Cl}(\operatorname{Int}(A)) \\
& \subset \operatorname{Int}(\operatorname{Cl}(A)) \cup \mathrm{Cl}(\operatorname{Int}(A)) \\
& \subset \operatorname{Int}(\mathrm{Cl}(W)) \cup \mathrm{Cl}(\operatorname{Int}(W)) .
\end{aligned}
$$

Therefore $W$ is $b$-open.

Corollary 4.24. If $A \subset W \subset p \mathrm{Cl}(A)$ and $A \in P \mathcal{I} O(X, \tau)$, then $W$ is b-open.

Proof. Let $A \in P \mathcal{I} O(X, \tau)$. Then by Theorem 4.4, $A \in \mathcal{I} O(X, \tau)$. Hence $W$ is $b$-open by Theorem 4.23.

Is every superset of a $P$ - $\mathcal{I}$-open set $P$ - $\mathcal{I}$-open? The answer is negative as seen in the example. 
Example 4.25. Consider the ideal topological space $(X, \tau, \mathcal{I})$ in Example 3.4. Let $A=\{b, c\}$ and $W=\{a, b, c\}$. Then $A$ is a $P$ - $\mathcal{I}$-open set and $A \subset W$. But $W$ is not a $P$ - $\mathcal{I}$-open set.

Theorem 4.26. Let $A$ be a subset of an ideal topological space $(X, \tau, \mathcal{I})$. If $A \subset W \subset p \operatorname{Int}\left(A_{p}^{*}\right)$ then $W$ is $P$ - $\mathcal{I}$-open.

Proof. Let $A$ be a subset of $X$. Suppose that $A \subset W \subset p \operatorname{Int}\left(A_{p}^{*}\right)$. Then $W \subset p \operatorname{Int}\left(A_{p}^{*}\right) \subset p \operatorname{Int}\left(W_{p}{ }^{*}\right)$ by Lemma 3.8. Hence $W$ is $P-\mathcal{I}$ open.

\section{5. $P-*$-closure in the ideal topological spaces}

Throughout this chapter $D(A)$ (resp. $\left.D_{\alpha}(A), D_{p}(A), D_{s}(A)\right)$ always mean a derived set (resp. $\alpha$-derived set, pre-derived set, semi-derived set) of $A$.

Definition 5.1. Let $(X, \tau, \mathcal{I})$ be an ideal topological space. Let $A$ be a subset of $X$. Then $\mathrm{Cl}_{p}^{*}(A, \tau, \mathcal{I})$ is defined as $\mathrm{Cl}_{p}^{*}(A, \tau, \mathcal{I})=A \cup A_{p}^{*}(\tau, \mathcal{I})$. $\mathrm{Cl}_{p}^{*}(A, \tau, \mathcal{I})$ is written simply as $\mathrm{Cl}_{p}^{*}(A)$ when there is no chance for confusion.

In an ideal topological space $(X, \tau, \mathcal{I}), \tau_{p}^{*}(\mathcal{I})$ is the collection of the complement of all sets satisfying the following condition :

$$
\mathrm{Cl}_{p}^{*}(A)=A \text { for } A \in \mathcal{P}(X)
$$

That is, $\tau_{p}^{*}(\mathcal{I}):=\left\{A^{c}: \mathrm{Cl}_{p}^{*}(A)=A\right.$ for $\left.A \in \mathcal{P}(X)\right\}$. $\tau_{p}^{*}(\mathcal{I})$ is written simply as $\tau_{p}^{*}$ when there is no chance for confusion.

Example 5.2. Consider the ideal topological space $(X, \tau, \mathcal{I})$ in Example 3.4. If $A=\{a, c\}$ then $A_{p}^{*}=\{c, d\}$. Hence $\mathrm{Cl}_{p}^{*}(A, \tau, \mathcal{I})=A \cup A_{p}^{*}=$ $\{a, c, d\}$.

Theorem 5.3. Let $(X, \tau, \mathcal{I})$ be an ideal topological space. Then the following assertions are valid.

(i) If $\mathcal{I}=\{\emptyset\}$ then $\mathrm{Cl}_{p}^{*}(A)=p \mathrm{Cl}(A)$.

(ii) If $\mathcal{I}=\mathcal{P}(X)$ then $\mathrm{Cl}_{p}^{*}(A)=A$.

(iii) If $A$ is $P$-I-open then $\mathrm{Cl}_{p}^{*}(A)=A_{p}^{*}$.

(iv) If $A \in \mathcal{I}$ then $\mathrm{Cl}_{p}^{*}(A)=A$.

Proof. Straightforward.

Let $(X, \tau, \mathcal{I})$ be an ideal topological space. If $A \subset W \subset \mathrm{Cl}_{p}^{*}(A)$ and $A \in P \mathcal{I} O(X, \tau)$, then is $W$ a $P$ - $\mathcal{I}$-open set? The answer to this question is negative as seen in the following example. 
Example 5.4. Let $X=\{a, b, c, d\}, \tau=\{X, \emptyset,\{c\},\{a, b\},\{a, b, c\}\}$ and $\mathcal{I}=\{\emptyset\}$. Then $(X, \tau, \mathcal{I})$ is an ideal topological space. Let $A=\{c\}$ and $W=\{c, d\}$. Then $A_{p}^{*}=\{c, d\}, \operatorname{Int}\left(A_{p}^{*}\right)=\{c\}$. We know that $A$ is $P$-I -open and $A \subset W \subset \mathrm{Cl}_{p}^{*}(A)$. But $W \not \subset \operatorname{Int}\left(W_{p}^{*}\right)=\{c\}$. Hence $W \notin P \mathcal{I} O(X, \tau)$.

Lemma 5.5. For any subsets $A$ and $B$ of an ideal topological space $(X, \tau, \mathcal{I})$, we have the following assertions:

(i) $\mathrm{Cl}_{p}^{*}(\emptyset)=\emptyset, \mathrm{Cl}_{p}^{*}(X)=X$.

(ii) If $A \subset B$ then $\mathrm{Cl}_{p}^{*}(A) \subset \mathrm{Cl}_{p}^{*}(B)$.

(iii) $\mathrm{Cl}_{p}^{*}\left(\mathrm{Cl}_{p}^{*}(A)\right) \supset \mathrm{Cl}_{p}^{*}(A)$.

(iv) $\mathrm{Cl}_{p}^{*}(A \cup B) \supset \mathrm{Cl}_{p}^{*}(A) \cup \mathrm{Cl}_{p}^{*}(B)$.

(v) $\mathrm{Cl}_{p}^{*}(A \cap B) \subset \mathrm{Cl}_{p}^{*}(A) \cap \mathrm{Cl}_{p}^{*}(B)$.

(vi) $\mathrm{Cl}_{p}^{*}(A) \subset \mathrm{Cl}^{*}(A)$.

(vii) $\mathrm{Cl}_{p}^{*}(A) \subset p \mathrm{Cl}(A)$.

(viii) Let $\mathcal{J}$ be a superset of $\mathcal{I}$. If $\mathcal{J}$ is an ideal, then $\mathrm{Cl}_{p}^{*}(A, \tau, \mathcal{I}) \supset$ $\mathrm{Cl}_{p}^{*}(A, \tau, \mathcal{J})$.

Proof. It is easy to verify the desired results by using Lemma 3.8, Theorem 3.5 and Definition 5.1.

In Lemma 5.5, the reverse inclusions of (iv), (v), (vi), (vii) and the converse of (ii) and (viii) are not valid as seen in the following example.

Example 5.6. Consider an ideal topological space $(X, \tau, \mathcal{I})$ where $X=\{a, b, c, d\}, \tau=\{X, \emptyset,\{c\},\{a, b\},\{a, b, c\}\}$ and $\mathcal{I}=\{\emptyset,\{c\}\}$. Let $A=\{a, d\}$ and $B=\{a, b\}$. Then $\mathrm{Cl}_{p}^{*}(A)=\{a, d\} \subset\{a, b, d\}=\mathrm{Cl}_{p}^{*}(B)$. But $A \not \subset B$. Let $A=\{a\}$ and $B=\{b\}$. Then $\mathrm{Cl}_{p}^{*}(A \cup B)=\{a, b, d\} \not \subset$ $\{a, b\}=\mathrm{Cl}_{p}^{*}(A) \cup \mathrm{Cl}_{p}^{*}(B)$. Let $A=\{a, d\}$ and $B=\{a, b\}$. Then $\mathrm{Cl}_{p}^{*}(A \cap$ $B)=\mathrm{Cl}_{p}^{*}(\{a\})=\{a\} \not \supset\{a, d\}=\mathrm{Cl}_{p}^{*}(A) \cap \mathrm{Cl}_{p}^{*}(B)$. Let $A=\{a, c\}$. Then $\mathrm{Cl}_{p}^{*}(A)=\{a, c\} \not \supset X=\mathrm{Cl}^{*}(A)$. Let $A=\{a, c\}$. Then $\mathrm{Cl}_{p}^{*}(A)=$ $\{a, c\} \not \supset\{a, c, d\}=p \operatorname{Cl}(A)$. Let $\mathcal{J}=\{\emptyset,\{a\}\}$. Then $\mathcal{J}$ is an ideal. Let $A=\{a, b\}$. Then $\mathrm{Cl}_{p}^{*}(A, \tau, \mathcal{I})=\{a, b, d\} \supset\{a, b\}=\mathrm{Cl}_{p}^{*}(A, \tau, \mathcal{J})$. But $\mathcal{I} \not \subset \mathcal{J}$.

In an ideal topological space $(X, \tau, \mathcal{I})$, since

$$
A \cup A_{p}^{*}=\mathrm{Cl}_{p}^{*}(A) \subset p \mathrm{Cl}(A)=A \cup D_{p}(A),
$$

we can guess that $A_{p}^{*} \subset D_{p}(A)$. But this is wrong as seen in the following example.

Example 5.7. Let $X=\{a, b, c, d\}, \tau=\{\emptyset, X,\{a, b\},\{a, b, c\}\}$ and $\mathcal{I}=\{\emptyset,\{a\}\}$. Then $(X, \tau, \mathcal{I})$ is an ideal topological space. If $A=$ 
$\{a, b, c\}$, then $A_{p}^{*}=\{b, c\}$ and $D_{p}(A)=\{c, d\}$. Hence $A_{p}^{*} \not \subset D_{p}(A)$ and $A_{p}^{*} \not \supset D_{p}(A)$.

Theorem 5.8. Let $(X, \tau, \mathcal{I})$ be an ideal topological space. For any subset $A$ of $X$, the following assertions are valid:

(i) $A_{p}^{*} \backslash A \subset p \mathrm{Cl}(A) \backslash A \subset D_{p}(A)$.

(ii) If $\mathcal{I}=\{\emptyset\}$, then $A_{p}^{*} \backslash A=p \operatorname{Cl}(A) \backslash A \subset D_{p}(A)$.

(iii) If $\mathcal{I}=\mathcal{P}(X)$, then $A_{p}^{*} \subset D_{p}(A)$.

Proof. (i) By Theorem 3.5, $A_{p}^{*} \subset p \mathrm{Cl}(A)$. Then $A_{p}^{*} \backslash A \subset p \mathrm{Cl}(A) \backslash A$. Since $p \operatorname{Cl}(A)=A \cup D_{p}(A), p \operatorname{Cl}(A) \backslash A \subset D_{p}(A)$. It follows that $A_{p}^{*} \backslash A \subset$ $p \operatorname{Cl}(A) \backslash A \subset D_{p}(A)$.

(ii) and (iii) are straightforward by (i) and Theorem 3.6.

Theorem 5.9. Let $(X, \tau, \mathcal{I})$ be an ideal topological space. If $\tau$ is a chain under the set inclusion, then either $\mathrm{Cl}_{p}^{*}(G)=X$ or $\mathrm{Cl}_{p}^{*}(G)=G$ for $G \subset X$.

Proof. It follows from Theorem 3.24.

Theorem 5.10. Let $(X, \tau, \mathcal{I})$ be an ideal topological space. Then $\tau^{p} \subset \tau_{p}^{*}$.

Proof. Let $A \in \tau^{p}$. Then $A^{c}$ is pre-closed (i.e. $A^{c}=p \mathrm{Cl}\left(A^{c}\right)$ ). This implies that $A^{c} \subset \mathrm{Cl}_{p}^{*}\left(A^{c}\right) \subset p \operatorname{Cl}\left(A^{c}\right)=A^{c}$. Hence $A^{c}=\mathrm{Cl}_{p}^{*}\left(A^{c}\right)$ (i.e. $A \in \tau_{p}^{*}$.) Therefore $\tau^{p} \subset \tau_{p}^{*}$.

Remark 5.11. The above theorem shows that every open set, preopen set, $P$ - $\mathcal{I}$-open set and $\mathcal{I}$-open set in an ideal topological space $(X, \tau, \mathcal{I})$ is a member of $\tau_{p}^{*}$.

The reverse inclusion of Theorem 5.10 may not hold as seen in the following example

Example 5.12. Let $X=\{a, b, c, d\}, \tau=\{\emptyset, X,\{a\},\{b, c\},\{a, b, c\}\}$ and $\mathcal{I}=\{\emptyset,\{c\}\}$. Then $(X, \tau, \mathcal{I})$ is an ideal topological space and

$\tau^{p}=\{\emptyset, X,\{a\},\{b\},\{c\},\{a, b\},\{a, c\},\{b, c\},\{a, b, c\},\{a, b, d\},\{a, c, d\}\}$

$\tau_{p}^{*}=\{\emptyset, X,\{a\},\{b\},\{c\},\{a, b\},\{a, c\},\{a, d\},\{b, c\},\{a, b, c\},\{a, b, d\}$, $\{a, c, d\}\}$. We know that $\{a, d\} \in \tau_{p}^{*}$ but $\{a, d\} \notin \tau_{p}$.

Theorem 5.13. Let $(X, \tau, \mathcal{I})$ be an ideal topological space. Then

(i) If $\mathcal{I}=\{\emptyset\}$ then $\tau_{p}^{*}=\tau^{p}$.

(ii) If $\mathcal{I}=\mathcal{P}(X)$ then $\tau_{p}^{*}=\mathcal{P}(X)$. 
Proof. (i) Since $\mathcal{I}=\{\emptyset\}, A_{p}^{*}=p \operatorname{Cl}(A)$ for all $A \in \mathcal{P}(X)$ by Theorem 3.6. Thus

$$
\begin{aligned}
\tau_{p}^{*} & =\left\{B^{c}: \mathrm{Cl}_{p}^{*}(B)=B \text { for } B \in \mathcal{P}(X)\right\} \\
& =\left\{B^{c}: B \cup B_{p}^{*}=B \text { for } B \in \mathcal{P}(X)\right\} \\
& =\left\{B^{c}: B \cup p \mathrm{Cl}(B)=B \text { for } B \in \mathcal{P}(X)\right\} \\
& =\left\{B^{c}: p \operatorname{Cl}(B)=B \text { for } B \in \mathcal{P}(X)\right\} \\
& =\tau^{p} .
\end{aligned}
$$

(ii) Suppose that $\mathcal{I}=\mathcal{P}(X)$. Let $A \in \mathcal{P}(X)$. Then $\mathrm{Cl}_{p}^{*}\left(A^{c}\right)=$ $A^{c} \cup\left(A^{c}\right)_{p}^{*}=A^{c}$ by Theorem 3.6. Hence $A \in \tau_{p}^{*}$. Therefore $\mathcal{P}(X) \subset \tau_{p}^{*}$, and hence $\mathcal{P}(X)=\tau_{p}^{*}$.

Theorem 5.14. Let $(X, \tau, \mathcal{I})$ be an ideal topological space. Then $\tau^{*} \subset \tau_{p}^{*}$.

Proof. Let $A \in \tau^{*}$. Then $\mathrm{Cl}^{*}\left(A^{c}\right)=A^{c}$, which implies that $A^{c} \subset$ $\mathrm{Cl}_{p}^{*}\left(A^{c}\right) \subset \mathrm{Cl}^{*}\left(A^{c}\right)=A^{c}$. Hence $\operatorname{Cl}_{p}^{*}\left(A^{c}\right)=A^{c}$ (i.e. $\left.A \in \tau_{p}^{*}\right)$. Therefore $\tau^{*} \subset \tau_{p}^{*}$.

The reverse inclusion of Theorem 5.14 does not hold as seen in the following example

Example 5.15. Let $X=\{a, b, c, d\}, \tau=\{\emptyset, X,\{c\},\{a, b\},\{a, b, c\}\}$ and $\mathcal{I}=\{\emptyset\}$. Then $(X, \tau, \mathcal{I})$ is an ideal topological space and

$$
\tau_{p}^{*}=\{\emptyset, X,\{a\},\{b\},\{c\},\{a, b\},\{a, c\},\{b, c\},\{a, b, c\},\{a, c, d\},\{b, c, d\}\}
$$

and

$$
\tau^{*}=\{\emptyset, X,\{c\},\{a, b\},\{a, b, c\}\} .
$$

We know that $\{b\} \in \tau_{p}^{*}$ but $\{b\} \notin \tau^{*}$.

Note that $\tau_{p}^{*}$ contains both $\tau^{p}$ and $\tau^{*}$. The following example shows that $\tau^{p}$ and $\tau^{*}$ are independent.

Example 5.16. Let $X=\{a, b, c, d\}, \tau=\{\emptyset, X,\{c\},\{a, b\},\{a, b, c\}\}$ and $\mathcal{I}=\{\emptyset,\{c\}\}$. Then $(X, \tau, \mathcal{I})$ is an ideal topological space and $\tau^{p}=\{\emptyset, X,\{a\},\{b\},\{c\},\{a, b\},\{a, c\},\{b, c\},\{a, b, c\},\{a, c, d\},\{b, c, d\}\}$ and

$$
\tau^{*}=\{\emptyset, X,\{c\},\{a, b\},\{a, b, c\},\{a, b, d\}\} .
$$

We know that $\tau^{p}$ and $\tau^{*}$ are independent.

Theorem 5.17. Let $(X, \tau, \mathcal{I})$ be an ideal topological space. If every member of $\tau$ is clopen then $\tau_{p}^{*}=\mathcal{P}(X)$. 
Proof. Let $A \in \mathcal{P}(X)$. Since every member of $\tau$ is clopen, $A \subset$ $\mathrm{Cl}(A)=\operatorname{Int}(\mathrm{Cl}(A))$ and so $A \in \tau^{p}$. This means that $\mathcal{P}(X)=\tau^{p}$. By Theorem 5.10, $\mathcal{P}(X)=\tau^{p} \subset \tau_{p}^{*} \subset \mathcal{P}(X)$. Hence $\tau_{p}^{*}=\mathcal{P}(X)$.

In an ideal topological space $(X, \tau, \mathcal{I})$, is $\tau_{p}^{*}$ a topology? The answer to this question is negative as seen in the following example.

Example 5.18. Consider the ideal topological space $(X, \tau, \mathcal{I})$ in Example 5.15. Then $\{a, c, d\},\{b, c, d\} \in \tau_{p}^{*}$. But $\{a, c, d\} \cap\{b, c, d\}=$ $\{c, d\} \notin \tau_{p}^{*}$.

Theorem 5.19. Let $(X, \tau, \mathcal{I})$ be an ideal topological space. If

$$
\mathrm{Cl}_{p}^{*}(B \cup C) \subset \mathrm{Cl}_{p}^{*}(B) \cup \mathrm{Cl}_{p}^{*}(C)
$$

for any subsets $B$ and $C$ of $X$, then $\tau_{p}^{*}$ is a topology.

Proof. Obviously, $\emptyset, X \in \tau_{p}^{*}$ by Lemma 5.5(i). Suppose that $G_{i} \in \tau_{p}^{*}$ for every $i \in \Lambda$. Then $\mathrm{Cl}_{p}^{*}\left(G_{i}^{c}\right)=G_{i}^{c}$ for every $i \in \Lambda$ and so

$$
\mathrm{Cl}_{p}^{*}\left(\left(\bigcup_{i \in \Lambda}\left(G_{i}\right)\right)^{c}\right)=\mathrm{Cl}_{p}^{*}\left(\bigcap_{i \in \Lambda} G_{i}^{c}\right) \subset \bigcap_{i \in \Lambda} \mathrm{Cl}_{p}^{*}\left(G_{i}^{c}\right)=\bigcap_{i \in \Lambda} G_{i}^{c}=\left(\bigcup_{i \in \Lambda} G_{i}\right)^{c} .
$$

Furthermore, $\left(\bigcup_{i \in \Lambda} G_{i}\right)^{c} \subset \mathrm{Cl}_{p}^{*}\left(\left(\bigcup_{i \in \Lambda} G_{i}\right)^{c}\right)$ by Definition 5.1. Hence

$$
\left(\bigcup_{i \in \Lambda} G_{i}\right)^{c}=\mathrm{Cl}_{p}^{*}\left(\left(\bigcup_{i \in \Lambda} G_{i}\right)^{c}\right)
$$

and so $\bigcup_{i \in \Lambda} G_{i} \in \tau_{p}^{*}$. Let $G, H \in \tau_{p}^{*}$. Then $\mathrm{Cl}_{p}^{*}\left(G^{c}\right)=G^{c}$ and $\mathrm{Cl}_{p}^{*}\left(H^{c}\right)=$ $H^{c}$. It follows from Lemma 5.5(iv) and hypothesis that $\mathrm{Cl}_{p}^{*}\left((G \cap H)^{c}\right)=\mathrm{Cl}_{p}^{*}\left(G^{c} \cup H^{c}\right)=\mathrm{Cl}_{p}^{*}\left(G^{c}\right) \cup \mathrm{Cl}_{p}^{*}\left(H^{c}\right)=G^{c} \cup H^{c}=(G \cap H)^{c}$ so that $G \cap H \in \tau_{p}^{*}$.

Corollary 5.20. Let $(X, \tau)$ be a topological space with ideal $\mathcal{I}=$ $\mathcal{P}(X)$. Then $\tau_{p}^{*}$ is a topology.

Proof. By Theorem 3.6(ii), $A_{p}^{*}=\emptyset$ for all subset $A$ of $X$. This implies that $\mathrm{Cl}_{p}^{*}(B \cup C)=(B \cup C) \cup(B \cup C)_{p}^{*}=B \cup C=\left(B \cup B_{p}^{*}\right) \cup\left(C \cup C_{p}^{*}\right)=$ $\mathrm{Cl}_{p}^{*}(B) \cup \mathrm{Cl}_{p}^{*}(C)$ for any subsets $B$ and $C$ of $X$. Hence by Theorem 5.19, $\tau_{p}^{*}$ is a topology.

Corollary 5.21. Let $(X, \tau, \mathcal{I})$ be an ideal topological space. If

$$
(B \cup C)_{p}^{*} \subset B_{p}^{*} \cup C_{p}^{*}
$$

for any subsets $B$ and $C$ of $X$, then $\tau_{p}^{*}$ is a topology. 
Proof. For any subsets $B$ and $C$ of $X,(B \cup C)_{p}^{*} \supset B_{p}^{*} \cup C_{p}^{*}$ by Lemma 3.8. Using hypothesis, we get $(B \cup C)_{p}^{*} \subset B_{p}^{*} \cup C_{p}^{*}$. Hence $(B \cup C)_{p}^{*}=$ $B_{p}^{*} \cup C_{p}^{*}$. we can easily check that $\mathrm{Cl}_{p}^{*}(B \cup C)=\mathrm{Cl}_{p}^{*}(B) \cup \mathrm{Cl}_{p}^{*}(C)$. It follows from Theorem 5.19 that $\tau_{p}^{*}$ is a topology.

Theorem 5.22. Let $(X, \tau, \mathcal{I})$ and $(X, \tau, \mathcal{J})$ be ideal topological spaces. If $\mathcal{I} \subset \mathcal{J}$ then $\tau_{p}^{*}(\mathcal{I}) \subset \tau_{p}^{*}(\mathcal{J})$.

Proof. Suppose that $\mathcal{I} \subset \mathcal{J}$ and let $A \in \tau_{p}^{*}(\mathcal{I})$. Using Lemma 5.5 (viii), we have $A^{c}=\mathrm{Cl}^{*}\left(A^{c}, \tau, \mathcal{I}\right) \supset \mathrm{Cl}^{*}\left(A^{c}, \tau, \mathcal{J}\right) \supset A^{c}$. Hence $\mathrm{Cl}^{*}\left(A^{c}, \tau, \mathcal{J}\right)=A^{c}$, i.e., $A \in \tau_{p}^{*}(\mathcal{J})$. Therefore $\tau_{p}^{*}(\mathcal{I}) \subset \tau_{p}^{*}(\mathcal{J})$.

Let $(X, \tau, \mathcal{I})$ and $(X, \kappa, \mathcal{I})$ be ideal topological spaces. If $\tau \subset \kappa$ then is the inclusion $\tau_{p}^{*} \subset \kappa_{p}^{*}$ valid? The answer is negative as seen in the following example.

Example 5.23. Consider two ideal topological spaces $(X, \tau, \mathcal{I})$ and $(X, \kappa, \mathcal{I})$ where $X=\{a, b, c, d\}, \tau=\{\emptyset, X,\{c\},\{a, b\},\{a, b, c\}\}, \kappa=\{\emptyset$, $X,\{a, b\},\{a, b, c\}\}$ and $\mathcal{I}=\{\emptyset\}$. Then

$$
\tau_{p}^{*}=\{\emptyset, X,\{a\},\{b\},\{c\},\{a, b\},\{a, c\},\{b, c\},\{a, b, c\},\{a, c, d\},\{b, c, d\}\}
$$

and $\kappa_{p}^{*}=\{\emptyset, X,\{a\},\{b\},\{a, b\},\{a, c\},\{a, d\},\{b, c\},\{b, d\},\{a, b, c\}$, $\{a, b, d\},\{a, c, d\},\{b, c, d\}\}$. It follows that $\tau_{p}^{*} \not \subset \kappa_{p}^{*}$ and $\tau_{p}^{*} \not \supset \kappa_{p}^{*}$.

Let $(X, \tau, \mathcal{I})$ be an ideal topological space. If $\mathcal{I} \subset \tau$ then is $\tau^{p}$ equal to $\tau_{p}^{*}$ ? The answer is negative as seen in the following example.

Example 5.24. Let $X=\{a, b, c, d\}, \tau=\{\emptyset, X,\{c\},\{a, b\},\{a, b, c\}\}$ and $\mathcal{I}=\{\emptyset,\{c\}\}$. Then $(X, \tau, \mathcal{I})$ is an ideal topological spaces with $\mathcal{I} \subset \tau$, and

$$
\tau^{p}=\{\emptyset, X,\{a\},\{b\},\{c\},\{a, b\},\{a, c\},\{b, c\},\{a, b, c\},\{a, c, d\},\{b, c, d\}\},
$$

and $\tau_{p}^{*}=\{\emptyset, X,\{a\},\{b\},\{c\},\{a, b\},\{a, c\},\{a, d\},\{b, c\},\{b, d\},\{a, b, c\}$, $\{a, b, d\},\{a, c, d\},\{b, c, d\}\}$. Clearly $\{a, d\} \in \tau_{p}^{*}$ but $\{a, d\} \notin \tau^{p}$. Hence $\tau^{p} \neq \tau_{p}^{*}$.

\section{References}

[1] A. Açikgöz, T. Noiri and Ş. Yüksel, On $\delta$-I-open sets and decomposition of $\alpha-I$ continuity, Acta Math. Hungar. 102 (2004), no. 4, 349-357.

[2] A. Açikgöz, T. Noiri and Ş. Yüksel, On $\alpha$-I-continuous and $\alpha$-I-open functions, Acta Math. Hungar. 105 (2004), no. 1-2, 27-37.

[3] D. Andrijevic, Some properties of the topology of $\alpha$-sets, Mat. Vesnik 36 (1984), $1-10$. 
[4] M. Akdağ, On b-I-open sets and b-I-continuous functions, Internat. J. Math. Math. Sci. 2007 Article ID 75721, 13 pages.

[5] M. Caldas, A note on some applications of $\alpha$-sets, Int. J. Math. Math. Sci. 2003 (2003), no. 2, 125-130.

[6] M. Caldas and J. Dontchev, On spaces with hereditarily compact $\alpha$-topologies, Acta Math. Hung. 82 (1999), 121-129.

[7] E. Hatir, A. Keskin and T. Noiri, A note on strong $\beta$-I-open sets and strongly $\beta$-I-continuous functions, Acta Math. Hungar. 108 (2005), no. 1-2, 87-94.

[8] E. Hatir and T. Noiri, On decompositions of continuity via idealization, Acta Math. Hungar. 96 (2002), no. 4, 341-349.

[9] E. Hatir and T. Noiri, Weakly pre-I-open sets and decomposition of continuity, Acta Math. Hungar. 106 (2005), no. 3, 227-238.

[10] E. Hatir and T. Noiri, On semi-I-open sets and semi-I-continuous functions, Acta Math. Hungar. 107 (2005), no. 4, 345-353.

[11] E. Hatir and T. Noiri, On $\beta$-I-open sets and a decomposition of almost-Icontinuity, Bull. Malay. Math. Sci. Soc. 29 (2006), no. 2, 119-124.

[12] S. Jafari and T. Noiri, Contra- $\alpha$-continuous functions between topological spaces, Iranian Int. J. Sci. 2 (2001), no. 2, 153-167.

[13] S. Jafari and T. Noiri, Some remarks on weak $\alpha$-continuity, Far East J. Math. Sci. 6 (1998), no. 4, 619-625.

[14] S. N. Maheshwari and S. S. Thakur, On $\alpha$-irresolute mappings, Tamkang J. Math. 11 (1980), 209-214.

[15] S. N. Maheshwari and S. S. Thakur, On $\alpha$-compact spaces, Bull. Inst. Math. Acad. Sinica 13 (1985), 341-347.

[16] H. Maki, R. Devi and K. Balachandran, Generalized $\alpha$-closed sets in topology, Bull. Fukuoka Univ. Ed. Part III 42 (1993), 13-21.

[17] H. Maki and T. Noiri, The pasting lemma for $\alpha$-continuous maps, Glas. Mat. 23(43) (1988), 357-363.

[18] A. S. Mashhour, I. A. Hasanein and S. N. El-Deeb, A note on semi-continuity and precontinuity, Indian J. Pure Appl. Math. 13 (1982), no. 10, 1119-1123.

[19] A. S. Mashhour, I. A. Hasanein and S. N. El-Deeb, $\alpha$-continuous and $\alpha$-open mappings, Acta Math. Hungar. 41 (1983), no. 3-4, 213-218.

[20] N. Levine, Semi-open sets and semi-continuity in topological spaces, Amer. Math. Monthly 70 (1963), 36-41.

[21] O. Njåstad, On some classes of nearly open sets, Pacific J. Math. 15 (1965), 961-970.

[22] I. L. Reilly and M. K. Vamanamurthy, On $\alpha$-sets in topological spaces, Tamkang J. Math. 16 (1985), 7-11.

[23] J. Tong, On decomposition of continuity in topological spaces, Acta Math. Hungar. 54 (1989), no. 1-2, 51-55.

[24] D. Andrijevic, On b-open sets, Mat. Vesnik 48, (1996), 59-64.

[25] D. Janković, and T.R. Hamlett, New topologies from old via ideals, Amer. Math. Monthly 97(4) (1990), 295-310.

[26] M.E. Abd El-Monsef, E.F. Lashien and A.A. Nasef, On I-open sets and Icontinious funtions, Kyungpook. Mathematicla. Journal 32 (1992), 21-30.

[27] Y. B. Jun, S, W. Jeong, H. J. Lee and J. W. Lee, Application of pre-open sets, Appl. General Top. 9 (2008), no. 2, 213-228. 
Department of Mathematics Education

Gyeongsang National University

Chinju 660-701, Korea

e-mail: 750-5095@hanmail.net (J. G. Kang)

e-mail: rnswk@chol.com (C. S. Kim) 